\title{
On the stability of best reply and gradient systems with applications to imperfectly competitive models
}

\author{
Luis C. Corchon ${ }^{\mathrm{a} . *}$, Andreu Mas-Colell ${ }^{\mathrm{b}}$ \\ ${ }^{a}$ Departamento de Fundamentos del Analisis Economico and Instituto Valenciano de Investigaciones Economicas, \\ Facultad de Economicas, Universidad de Alicante, 03071 Alicante, Spain \\ ${ }^{b}$ Department of Economics, Harvard University, Cambridge, MA 02138, USA
}

Received 21 June 1995; accepted 21 July 1995

\begin{abstract}
We present a general result on the convergence to an equilibrium of a class of dynamic adjustment procedures which includes gradient systems and best reply dynamics as special cases - when there are two players and strategy sets are one dimensional. We also show that there are no restrictions on the form of the gradient or best reply dynamics, even under strong restrictions on the functional form of both demand and costs. This implies that we can construct examples with three players where the above dynamical procedures yield chaotic behavior.
\end{abstract}

Keywords: Gradient; Best reply; Chaos; Stability

JEL classification: $\mathrm{C} 73$

\section{Introduction}

The origins of the theory of dynamic stability in imperfectly competitive economies can be traced to Cournot (1897). He defined what in modern terms is called the best reply dynamics and conjectured that, in the case of oligopoly with homogeneous goods, an equilibrium would be reached asymptotically. The modern literature has pursued two different approaches to the study of best reply dynamics, based on discrete time and on continuous time, respectively. Contributions in the former framework are Friedman (1977), Moulin (1982), Lippman et al. (1987), Vives (1990) and Milgrom and Roberts (1990). Their results imply that under some (strong) assumptions, best reply dynamics (and mild variations of it) converge asymptotically

\footnotetext{
* Corresponding author.
} 
to an equilibrium. A fulfilled expectations variation of best reply dynamics, initially proposed by Maskin and Tirole $(1987)$, has been studied by Dana and Montrucchio $(1986,1987)$ for the case of two players. They found that in general the possibility of chaotic behavior cannot be precluded. In the continuous time framework and assuming differential adjustments, Hahn (1962) and Okuguchi (1964) showed that with a homogeneous produce, strategic substitution (i.e. the best reply function of any player is decreasing in the strategy of competitors) and an additional condition on the slopes of the inverse demand and marginal cost functions, the best reply dynamics converges asymptotically to the (unique) Cournot equilibrium. In a general equilibrium model, Negishi (1961) proved convergence of a related dynamic system (see also Hirsch and Smale, 1974, pp. 265-273; Gaunersdorfer and Hofbauer, 1994). One advantage of the continuous approach over the discrete time approach is that any possible instabilities of the system are not linked to overshooting phenomena, a type of instability that is important but which may be better considered separately.

We attempt to answer the following question. What can be said about stability in continuous time that does not depend on special assumptions (beyond that strategy sets are one dimensional) about the underlying economy? In fact, we study this matter for gradient dynamics (see Arrow et al., 1958) which, for the theory that concerns us, can be shown to include the results on best reply dynamics as a special case. We first tackle the case of two players. By adapting an argument of Keenan and Rader (1985), based on Liouville's theorem, we are able to show that, if the strategy space is one dimensional, then convergence of a class of dynamic processes that includes gradient and best reply dynamics systems obtains with considerable generality (Proposition 1). We then move to a deeper investigation into the properties of the functional form of gradient and best reply dynamics in oligopoly models. Our next result shows that this functional form is essentially arbitrary, even under strong a priori restrictions on the shape of the demand and cost functions. Specifically, assuming that firms are price-setters we show that any list of $n$ arbitrary functions can be rationalized as gradient dynamics of $n$ firms with zero costs (or, in general, identical and constant marginal costs) and a system of demand functions with a negative dominant diagonal Jacobian (Proposition 2). An identical result can be proved for the case of best reply dynamics (Proposition 3). Similar results hold when firms are quantity-setters. Propositions 2 and 3 are reminiscent of the results obtained by Sonnenschein (1972), Mantel (1974), and Debreu (1974) on the characterization of excess demand functions in the perfectly competitive case (or of Boldrin and Montrucchio, 1986, for equilibrium dynamics decision rules). However, we should emphasize that our results (like those of Boldrin and Montrucchio) are obtained under restrictions on the class of allowable demand and cost functions which, for the perfectly competitive case, would imply that the equilibrium is unique and globally tatonement stable. A corollary of these results is that we can find an economy with three identical firms with constant marginal costs and a system of demand functions with a negative dominant diagonal Jacobian such that its gradient (or best reply) dynamics yield chaotic behavior.

\footnotetext{
'This mathematical result has also been used in evolutionary game theory, see, for example, Hofbauer and Sigmund (1988).
} 


\section{The model}

There are $n$ players. Each player $i$ has a one-dimensional strategy set $S_{i}=\left[a_{i}, b_{i}\right] \subset \mathbb{R}_{+}$, with a typical element $s_{i}$. Let $S=\mathrm{X}_{i=1}^{n} S_{i}$. As is customary, for any $i$ we also express the strategies profiles $s \in S$ as $s=\left(s_{i}, s_{-i}\right){ }^{2}$

In this paper we are concerned with autonomous dynamical systems of the following form:

$$
\dot{s}_{i}=G_{i}(s), \quad i=1,2, \ldots, n
$$

or, in a more compact notation:

$$
\dot{s}=G(s) .
$$

An equilibrium of (1) is an $s^{*} \in S$ such that $G\left(s^{*}\right)=0$. let $D G(s)$ be the Jacobian of $G(\cdot)$, evaluated at $s$, and $\operatorname{Tr}(D G(s))$ the trace of $D G(s)$, i.e. the sum of all the diagonal elements. We assume the following properties:

(D) $G_{i}(\cdot)$ is a continuously differentiable function, for every $i$.

(N) $\operatorname{Tr}(D G(s))$ is negative for all $s \in S$.

(B) For every $s \in S$ and $i$ we have: if $s_{i}=a_{i}$, then $G_{i}(s) \geqslant 0$; if $s_{i}=b_{i}$, then $G_{i}(s) \leqslant 0$.

Two natural examples (for a suitable choice of $a_{i}$ and $b_{i}$ ) the satisfy properties (D), (N) and (B) are:

(1) Best reply dynamics: $\dot{s}_{i}=\mu_{i}\left(\mathrm{BRF}_{i}\left(s_{-i}\right)-s_{i}\right), \mu_{i}>0$, for every $i$, where $\mathrm{BRF}_{i}(\cdot)$ stands for the (smooth) best reply function of player $i$ and $\mu_{i}$ is the speed of adjustment of strategy $i$.

(2) Gradient dynamics: $\dot{s}_{i}=\mu_{i}\left(\partial u_{i}(s) / \partial s_{i}\right), \mu_{i}>0$, for every $i$, where $u_{i}(s)$ is the utility payoff of player $i$, assumed differentiably strictly concave in its own strategy, $s_{i}$. Notice that best reply dynamics is observationally indistinguishable from the gradient dynamics generated by the 'fictitious' utility function $\hat{u}_{i}(s)=s_{i} \operatorname{BRF}_{i}\left(s_{-i}\right)-s_{i}^{2} / 2$ since $\partial \hat{u}_{i}(s) / \partial s_{i}=\operatorname{BRF}_{i}\left(s_{-i}\right)-s_{i}$.

Finally, assume the following regularity condition:

(T) The determinant of $D G(s)$, denoted by $|D G(s)|$, is non-vanishing at any equilibrium $s^{*}$.

Let $\phi_{t}(\bar{s})$ be the position of system (1) at time $t$ as a function of $\bar{s}$, an initial point in $S$. We call $\phi_{t}(\bar{s})$ a trajectory. We are now ready to prove our first result:

\footnotetext{
${ }^{2}$ The hypothesis that strategy sets are one dimensional when combined with an assumption that best replies are unique is akin to a requirement of single peakedness in payoff functions (see Krishna, 1992) for the use of single peakedness in the analysis of adjustment dynamics in games.
} 
Proposition 1. If $n=2$, then, under the hypotheses made, any trajectory $\phi_{t}(\bar{s})$ converges to some equilibrium.

Proof. To keep things simple we take the boundary condition to hold strictly. Let Vol $E$ denote the volume (area in our case) of any region $E \subset S$. By Liouville's theorem (see Arnold, 1973, p. 198; a nice and self-contained proof of the Liouville theorem can be found in Keenan and Rader, 1985, pp. 467-468) the fact that $\operatorname{Tr}(D G(s))<0$ implies that $G$ contracts the volume, i.e. for any region $E \subset S$ with $\operatorname{Vol} E>0$ and for any $t>0$ we have that $\operatorname{Vol}\left(\phi_{t}(E)\right)<$ Vol $E$.

Now suppose that from some initial point $\bar{s}$ the trajectory $\phi_{t}(\bar{s})$ does not converge to an equilibrium. The situation must then be as in Fig. 1. That is, the trajectory spirals around a limit set which is formed by a finite number of $M$ (possible $M=0$ ) equilibria, $s_{1}, \ldots, s_{M}$, with $s_{1}=s_{M}$, and of trajectories, $\mu_{1}, \ldots, \mu_{M}$, such that $\mu_{r}$ connects $s_{r}$ with $s_{r+1}$ (if $M=0$, then the limit set is a limit cycle; see Lefschetz, 1946, p. 172). Under the above conditions the limit set contains a (topological) circle (it may contain more) for which its interior $E$ is invariant and has $\operatorname{Vol} E>0$ (see Fig. 1). Hence, for $t>0, \operatorname{Vol}\left(\phi_{t}(E)\right)=\operatorname{Vol} E$, which contradicts the hypothesis.

Our assumptions are compatible with multiple equilibria. Notice, however, that (N) imposes a restriction on the nature of these equilibria: if $G(s)=0$, then $s$ is either a sink (if $|D G(s)|>0$ ) or a saddle (if $|D G(s)|<0$ ), but never a source. Thus Fig. 2 offers a simple representation of the dynamics generated by (1).

Consider for a moment the gradient dynamics. If we were dealing with a zero-sum game,

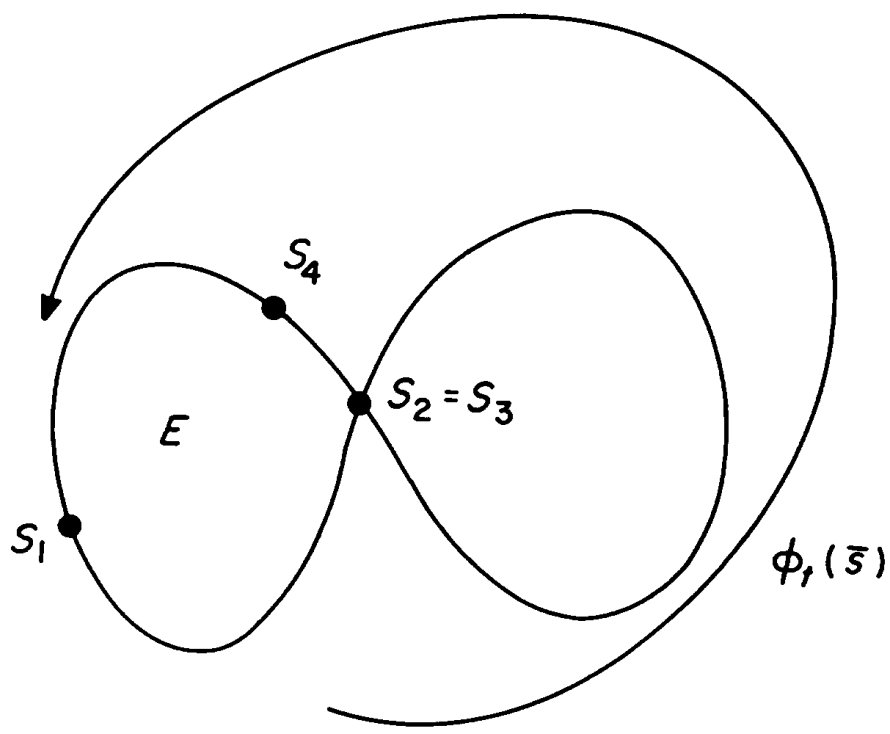

Fig. 1. 


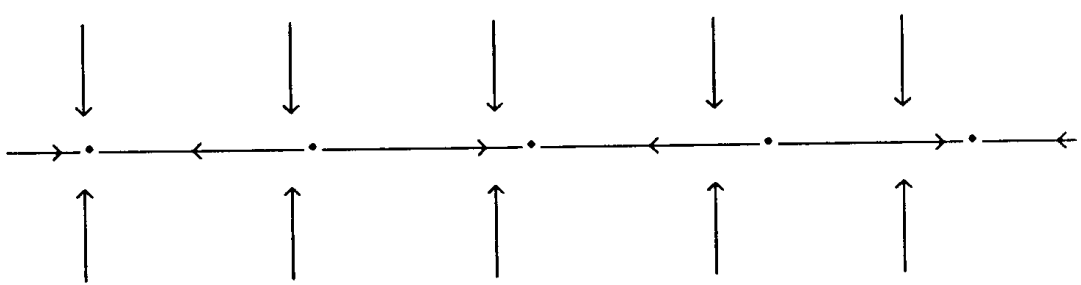

Fig. 2.

that is, if $u_{1}(s)+u_{2}(s)=0$ for all $s \in S$, then it is known that under our hypothesis (including, among these, the concavity of payoff functions with respect to own strategies) there is a single equilibrium, which is a globally convergent saddle (see Arrow et al., 1958). Thus, for the special case of one-dimensional strategies, Proposition 1 can be viewed as a generalization of the convergence part of this classical result.

Next we present a general method that allows us to find the economy that generates a given dynamical system. In fact, to show that our results do not merely reproduce those of Sonnenschein (1972), Mantel (1972) and Debreu (1974) on the characterization of excess demand functions, we construct such an economy from a very restricted set of economies.

Definition. The set of nice economies (NE in what follows) is composed of economies displaying the following characteristics:

(1) There are $n$ firms that produce a differentiated product each from a unique, nonproduced input (e.g. labor).

(2) Firms have identical and constant marginal costs.

(3) The system of demand functions has a negative dominant diagonal, i.e. if $X_{i}(p)$ is the demand function of good $i$, for all $p \gg 0$ we have that $\left(\partial X_{i}(p) / \partial p_{i}\right)<0$ and $\left|\left(\partial X_{i}(p) / \partial p_{i}\right)\right|>$ $\left.\sum_{j \neq i} \mid \partial X_{i}(p) / \partial p_{j}\right) \mid, i=1, \ldots, n$.

We remark that any economy that belongs to the set NE has a unique and globally stable Walrasian equilibrium (see, for example, Arrow and Hahn, 1971). However, when oligopolistic competition is the relevant solution concept, the situation is dramatically different, as is made clear by the following proposition.

Proposition 2. Let $\dot{p}_{i}=G_{i}\left(p_{1}, \ldots, p_{i}, \ldots, p_{n}\right)$, with $p_{i} \in\left[a_{i}, b_{i}\right]\left(a_{i}>0\right), i=1, \ldots, n$, be a given differentiable dynamical system with $\partial G_{i} / \partial p_{i}<0$ for all $i$. Then there exists an economy from the set $N E$ which generates the above equations as a gradient system.

Proof. We assume costs to be zero (a similar construction to the one below applies if costs were required to be positive). Let us consider $G_{i}\left(p_{1}, \ldots, p_{i}, \ldots, p_{n}\right)$. For a given $p_{-i}$ this function is integrable in $p_{i}$. Let $F_{i}\left(p_{1}, \ldots, p_{i}, \ldots, p_{n}\right) \equiv \int_{a_{i}}^{p_{i}} G_{i}\left(s, p_{-i}\right)$ ds. Then the demand function for firm $i$ is $X_{i}=\left(F_{i}\left(p_{1}, \ldots, p_{i}, \ldots, p_{n}\right)+b\right) / p_{i}$, where $b>0$. Notice that taking $b$ large enough, demand is positive for every price and the dominant diagonal property holds since $\Sigma_{i \neq i} \partial X_{i}(p) / \partial p$, is bounded and independent of $b$, but $\left(\partial X_{i}(p) / \partial p_{i}\right)$ depends monotoni- 
cally on $b$ and is as large, as we wish. Finally, profits for firm $i$ are $\pi_{i}=X_{i} p_{i}=$ $F_{i}\left(p_{1}, \ldots, p_{i}, \ldots, p_{n}\right)+b$. Clearly, $\partial \pi_{i}(p) / \partial p_{i}=G_{i}\left(p_{1}, \ldots, p_{i}, \ldots, p_{n}\right)$.

The next proposition considers best reply dynamics.

Proposition 3. Let $\dot{p}_{i}=f_{i}\left(p_{-i}\right)-p_{i}$, with $p_{i} \in\left[a_{i}, b_{i}\right]\left(a_{i}>0\right), i=1, \ldots, n$, be a given differentiable dynamical system. Then there exists an economy from the set $N E$ which generates the above equations as best reply dynamics.

Proof. Using the construction given in the proof of Proposition 2 for a system of demand functions of the form $X_{i}=f_{i}\left(p_{-i}\right)-p_{i} / 2+b / p_{i}$ produces the desired result.

Propositions 2 and 3 can be proved for the case of quantity-setting firms with straightforward modifications. An implication of Propositions 2 and 3 is that in the case of three firms we can find gradient (or best reply) dynamical systems such that they yield chaotic behavior (e.g. Lorenz's equations; see Guckenheimer and Holmes, 1983, p. 93ff.). Details can be obtained from the authors on request.

\section{Acknowledgements}

We thank Rose-Anne Dana, Drew Fudenberg, Paco Marhuenda, Luigi Montrucchio and audiences at Austin (Texas), Caltech and Duke University of North Carolina at Chapel Hill for comments and suggestions. The first author acknowledges financial support from Generalitat Valenciana, DGICYT, under Project PB91/0756, and the hospitality of the Royal Complutense College at Harvard.

\section{References}

Arnold, V. I., 1973, Ordinary differential equations (MIT Press, Cambridge, MA).

Arrow, K. and F.H. Hahn, 1971, General competitive analysis (Holden Day, San Francisco, CA).

Arrow, K., L. Hurwicz and H. Uzawa, eds., 1958, Studies in linear and non linear programming (Stanford University Press, Stanford, CA).

Boldrin, M. and L. Montrucchio, 1986, On the indeterminacy of capital accumulation paths, Journal of Economic Theory 40, no. $1,26-39$.

Cournot, A.A., 1897, Researches into the mathematical principles of the theory of wealth (MacMillan, New York).

Dana, R.A. and L. Montrucchio, 1986, Dynamic complexity in duopoly games, Journal of Economic Theory 40 , no. $1,40-56$.

Dana, R.A. and L. Montrucchio, 1987, On rational dynamic strategies in infinite horizon models where agents discount the future, Journal of Economic Behavior and Organization 8, 497-511.

Debreu, G., 1974, Excess demand functions, Journal of Mathematical Economics 1, 15-21.

Friedman, J., 1977, Oligopoly and theory of games (North-Holland, Amsterdam).

Gaunersdorfer, A. and J. Hofbauer, 1994, Fictitious play, Shapley polygons, and the replicator equation, Mimeo, Vienna. 
Guckenheimer, J. and P. Holmes, 1983, Nonlinear oscillations, dynamical systems, and bifurcations of vector fields, Applied Mathematical Sciences 42 (Springer, Berlin).

Hahn, F.H., 1962, The stability of the Cournot oligopoly solution, Review of Economic Studies 29, no. 4, 329-333.

Hirsch, M.W. and S. Smale, 1974, Different equations, dynamical systems and linear algebra (Academic Press, New York).

Hofbauer, J. and K. Sigmund, 1988, The theory of evolution and dynamical systems (Cambridge University Press, Cambridge).

Keenan, D. and T. Rader, 1985, Market dynamics and the law of demand, Econometrica 53, no. 2, $465-471$.

Krishna, V., 1992, Learning in games with strategic complementarities, Working Paper 92-073, Harvard Business School, Cambridge, MA.

Lefschetz, S., 1946, Lectures on differential equations (Princeton University Press, Princeton, NJ).

Lippman, S.A., J.W. Mamer and K.F. McCardle, 1987, Comparative statics in non-cooperative games via transinfinitely iterated play, Journal of Economic Theory 41, 288-303.

Mantel, R., 1974, On the characterization of aggregate excess demand, Journal of Economic Theory 7, 348-353.

Maskin, E. and J. Tirole, 1987, A theory of dynamic oligopoly III, European Economic Review 31, $947-968$.

Milgrom, P. and J. Roberts, 1990, Rationalizability, learning and equilibrium in games with strategic complementarities, Econometrica 58, no. 6, 1255-1277.

Moulin, H., 1982, Game theory for the social sciences (New York University Press, New York).

Negishi, T., 1961, Monopolistic competition and general equilibrium, Review of Economic Studies 28, 196-201.

Okuguchi, K., 1964, The stability of the Cournot oligopoly solution: A further generalization, Review of Economic Studies 31, no. 2, 143-146.

Sonnenschein, H., 1972, Market excess demand functions, Econometrica 40, 549-563.

Vives, X., 1990, Nash equilibrium and strategic complementarities, Journal of Mathematical Economics 19 , $305-321$. 